\title{
Outcomes of first-line osimertinib treatment for epidermal growth factor receptor-mutated non- small cell lung cancer in clinical practice settings
}

Toshihiro Shiozawa ( $\nabla$ t-shiozawa@md.tsukuba.ac.jp )

University of Tsukuba

Takeshi Numata

National Hospital Organization Mito Medical Center

Tomohiro Tamura

Ibaraki Prefectural Central Hospital

Takeo Endo

National Hospital Organization Mito Medical Center

Takayuki Kaburagi

Ibaraki Prefectural Central Hospital

Yusuke Yamamoto

Hitachi General Hospital

Hideyasu Yamada

Hitachinaka General Hospital

Norihiro Kikuchi

Kasumigaura Medical Center

Kazuhito Saito

Tsuchiura Kyodo General Hospital

Masaharu Inagaki

Tsuchiura Kyodo General Hospital

Koichi Kurishima

Tsukuba Medical Center Hospital

Yasunori Funayama

Tsukuba Gakuen Hospital

Kunihiko Miyazaki

Ryugasaki Saiseikai Hospital

Nobuyuki Koyama

Saitama Medical University

Kinya Furukawa

Tokyo Medical University Ibaraki Medical Center

Hiroyuki Nakamura 
Tokyo Medical University Ibaraki Medical Center

\section{Shinji Kikuchi}

University of Tsukuba

\section{Hideo Ichimura}

University of Tsukuba

\section{Yukio Sato}

University of Tsukuba

Ikuo Sekine

University of Tsukuba

Hiroaki Satoh

University of Tsukuba

Nobuyuki Hizawa

University of Tsukuba

\section{Short Report}

Keywords: lung cancer, EGFR mutation, osimertinib, PD-L1

Posted Date: January 20th, 2022

DOI: https://doi.org/10.21203/rs.3.rs-1272469/v1

License: (c) (i) This work is licensed under a Creative Commons Attribution 4.0 International License. Read Full License 
Outcomes of first-line osimertinib treatment for epidermal growth factor receptor-mutated nonsmall cell lung cancer in clinical practice settings

Toshihiro Shiozawa ${ }^{1 *}$; Takeshi Numata ${ }^{2 *}$; Tomohiro Tamura ${ }^{3}$; Takeo Endo ${ }^{2}$; Takayuki Kaburagi ${ }^{3}$; Yusuke Yamamoto $^{4}$; Hideyasu Yamada ${ }^{5}$ N Norihiro Kikuchi ${ }^{6}$; Kazuhito Saito ${ }^{7}$ Masaharu Inagaki ${ }^{7}$; Koichi Kurishima ${ }^{8}$; Yasunori Funayama; Kunihiko Miyazaki ${ }^{10}$; Nobuyuki Koyama ${ }^{11}$; Kinya Furukawa ${ }^{12}$; Hiroyuki Nakamura $^{12}$; Shinji Kikuchi ${ }^{13}$; Hideo Ichimura ${ }^{13}$; Yukio Sato ${ }^{13}$; Ikuo Sekine ${ }^{14}$; Hiroaki Satoh ${ }^{15}$; Nobuyuki Hizawa ${ }^{1}$

*Toshihiro Shiozawa and Takeshi Numata should be considered joint first author

Affiliations:

1. Department of Respiratory Medicine, Faculty of Medicine, University of Tsukuba, Tsukuba, Japan

2. Divisions of Respiratory Medicine, Mito Medical Center, Mito, Japan

3. Respiratory Center, Ibaraki Prefectural Central Hospital, Kasama, Japan

4. Divisions of Respiratory Medicine, Hitachi General Hospital, Hitachi, Japan

5. Division of Respiratory Medicine, Hitachinaka Medical Center, University of Tsukuba, Hitachinaka, Japan

6. Division of Respiratory Medicine, Kasumigaura Medical Center, Tsuchiura, Japan 
7. Divisions of Respiratory Medicine and Thoracic Surgery, Tsuchiura Kyodo General Hospital, Tsuchiura, Japan

8. Division of Respiratory Medicine, Tsukuba Medical Center Hospital, Tsukuba, Ibaraki, Japan

9. Division of Respiratory Medicine, Tsukuba Gakuen Hospital, Tsukuba, Japan

10. Division of Respiratory Medicine, Ryugasaki Saiseikai Hospital, Ryugasaki, Japan

11. Department of Respiratory Medicine, Saitama Medical Center, Saitama Medical University, Saitama, Japan

12. Divisions of Respiratory Medicine and Thoracic Surgery, Tokyo Medical University, Ibaraki Medical Center, Ami, Japan

13. Department of Thoracic Surgery, Faculty of Medicine, University of Tsukuba, Tsukuba, Japan

14. Department of Medical Oncology, Faculty of Medicine, University of Tsukuba, Tsukuba, Japan

15. Division of Respiratory Medicine, Mito Kyodo General Hospital-Mito Medical Center, University of Tsukuba, Mito, Japan

\section{Corresponding author:}

Toshihiro Shiozawa, M.D.

Department of Respiratory Medicine, Faculty of Medicine, University of Tsukuba

1-1-1 Tennoudai, Tsukuba, Ibaraki 305-8575, Japan 
E-mail: t-shiozawa@md.tsukuba.ac.jp

Fax: +81-29-853-3144

Phone: +81-29-853-3144 


\section{Abstract}

Background: Real-world data on the clinical outcomes of first-line osimertinib treatment for non-small cell lung cancer (NSCLC) with epidermal growth factor receptor (EGFR) mutations is lacking. This study aimed to evaluate the treatment outcomes and the prognostic factors of osimertinib as first-line therapy in clinical practice settings.

Methods: We retrospectively evaluated clinical outcomes of patients with $E G F R$-mutated NSCLC treated with osimertinib as first-line therapy across 12 institutions in Japan between August 2018 and March 2020.

Results: Among 158 enrolled patients, the objective response rate (ORR) was $68 \%$ and the estimated median progression-free survival (PFS) was 17.1 months (95\% confidence interval [CI], 14.5-19.7). Subgroup analysis showed that PFS in the group with high programmed death-ligand 1 (PD-L1) expression was significantly shorter than that in groups with low or no PD-L1 expression (10.1 vs. 16.1 vs. 19.0 months; $p=0.03$ ). Univariate and multivariate analyses demonstrated that high PD-L1 expression was the only independent adverse prognostic factor of osimertinib outcome for PFS (hazard ratio, 2.71; 95\% CI: 1.26-5.84; $p=0.01)$. In terms of the ORR, there was no statistically significant difference regardless of PDL1 expression ( $67 \%$ vs. $76 \%$ vs. $65 \% ; p=0.51)$.

Conclusion: Osimertinib showed favorable treatment outcomes in clinical practice. Although the ORR was similar regardless of PD-L1 expression, high PD-L1 expression could be an independent adverse prognostic factor related to PFS in osimertinib treatment. 
Keywords

lung cancer, EGFR mutation, osimertinib, PD-L1 


\section{Introduction}

Molecularly targeted therapies have contributed to an improvement in the survival of patients with recurrent or advanced non-small cell lung cancer (NSCLC) harboring driver oncogenes. Mutations in the epidermal growth factor receptor $(E G F R)$, which is a driver oncogene in NSCLC, lead to tumorigenesis and tumor growth via the activated EGFR-signaling pathway [1]. Previous phase III studies demonstrated that EGFR tyrosine kinase inhibitors (EGFR-TKIs) as first-line therapy for EGFR-mutated NSCLC had better outcomes than a platinum-based regimen in terms of both progression-free survival (PFS) and objective response rate (ORR) [2-4]. EGFR-TKIs are thus the current standard first-line agents for treating patients with $E G F R$-mutated advanced NSCLC.

First- to third-generation EGFR-TKIs are available in clinical practice. Of these, osimertinib, categorized as a third-generation EGFR-TKI, has irreversible anti-tumor activity against both EGFRsensitizing and EGFR-resistant T790M mutations. In the global phase III FLAURA trial involving patients with untreated EGFR-mutated recurrent or advanced NSCLC, osimertinib prolonged PFS and overall survival (OS) compared with the standard of care achieved by first-generation EGFR-TKIs [5, 6]. Thus, osimertinib is regarded as the most recommended first-line agent in these patients [7].

In clinical practice, osimertinib is indicated for a heterogeneous population, including patients with decreased performance status, symptomatic brain or leptomeningeal metastases, and uncommon mutations. However, as per the criteria of FLAURA trials, these patient groups are ineligible for osimertinib 
treatment. This discrepancy in recommendations suggests that there is a data gap regarding treatment outcomes between results of the FLAURA trial and those noted in current clinical practice. Therefore, in addition to pivotal clinical trial data, it is important to collect and analyze post-marketing clinical data. Although the use of osimertinib as a first-line agent has increased since its approval, data regarding outcomes and the prognostic factors with this treatment in clinical practice are still lacking.

To bridge this knowledge gap, we conducted this multi-institutional, retrospective, observational study to evaluate the treatment outcomes and the prognostic factors of first-line osimertinib for treatment of patients with recurrent or advanced $E G F R$-mutated NSCLC.

\section{Patients and methods}

\section{Data collection}

Twelve institutions in Ibaraki Prefecture, Japan participated in this study. We enrolled patients with recurrent or advanced NSCLC with EGFR mutations who received osimertinib as a first-line agent between August 2018 and March 2020. The data cut-off was May 31, 2020.

The following clinical data were collected: age, sex, smoking status (current, former, or never), Eastern Cooperative Oncology Group performance status (PS), stage at diagnosis according to the TNM Classification of Malignant Tumors (eighth edition), histology, type of EGFR mutation, presence of central nervous system (CNS) metastasis, and programmed death-ligand 1 (PD-L1) expression status using 
immunohistochemistry. Based on previous studies, PD-L1 expression was classified as none, low, and high

if the tumor percentage score (TPS) of PD-L1 was $<1 \%, 1 \%-49 \%$, and $>50 \%$, respectively $[8,9]$.

\section{Statistical analysis}

The endpoints in this study were the efficacy outcomes. The radiological anti-tumor response was evaluated based on Response Evaluation Criteria in Solid Tumors (version 1.0.10). The ORR was defined as the proportion of patients who achieved anti-tumor response with complete response (CR) or partial response (PR). Disease control rate (DCR) was defined as the ORR plus the proportion of patients achieving stable disease (SD).

In this study, PFS was defined as the duration from the initiation of osimertinib treatment to disease progression or death from any cause. OS was defined as the duration from the initiation of osimertinib treatment to death from any cause. If death did not occur at the cut-off date, patients were censored. If patients were lost during the observation period, they were censored on the last day of confirmed survival. Clinical evaluations of PFS and OS were conducted using the Kaplan-Meier method. Log-rank test was used to compare two different survival curves. A Cox regression model was applied to examine prognostic factors related to survival. Univariate and multivariate hazard ratios (HRs) were reported with $95 \%$ confidence intervals (CIs). Statistical analysis was performed using IBM SPSS Statistics for Windows (version 24.0; IBM Corp., Armonk, NY, USA). All tests were two-sided and were judged 
statistically significant if the calculated $p$-values were $<0.05$.

\section{Results}

Patient characteristics

Among 161 patients initially enrolled, three were excluded from the analysis owing to the lack of data,

resulting in a total of 158 eligible patients for the current study. Table 1 shows the patient characteristics.

The median age was 73 (range, 39-93) years, females accounted for $58 \%$ of the sample population, and adenocarcinoma was present in $95 \%$ of all cases. The proportions of EGFR mutation subtypes were $52 \%$ exon19 deletion, 43\% exon 21 L858R point mutation, and 5\% uncommon mutation. Forty-five patients (28\%) had CNS metastases at the time of diagnosis. The TPS of PD-L1 was none, low, and high in 60 (38\%), 41 (26\%), and 27 (17\%) patients, respectively. The remaining 30 patients (19\%) had unknown TPS.

Survival

The median follow-up period of the present study was 12.5 months. The estimated median PFS was 17.1

months $(95 \% \mathrm{CI}, 14.5-19.7)$. OS did not reach the median.

We examined the outcomes of osimertinib therapy according to patient subgroups of age, sex, smoking status, PS, stage, mutation subtype, CNS metastases, and TPS. No significant difference in PFS was observed with respect to age ( $<70$ vs. $\geq 70$ years, Fig. 1a), sex (male vs. female, Fig. 1b), smoking 
status (never vs. current or former, Fig. 1c), PS (0-1 vs. 2-4, Fig. 1d), stage (III or recurrent vs. IV, Fig. 1e), mutation subtype (Exon 19 del vs. L858R vs. uncommon, Fig. 1f), and CNS metastasis (present vs. absent Fig. 1g). However, the high TPS group had a significantly poorer PFS of 10.1 months compared with the low and no TPS groups that had 16.1 and 19.0 months, respectively (log-rank $p=0.03$, Fig. 1h).

Next, we performed univariate and multivariate analyses to evaluate prognostic factors associated with PFS. Among collected patient characteristics, we selected the eight factors mentioned above. As shown in Table 2, only high TPS was found to be a statistically significant adverse prognostic factor related to PFS $(\mathrm{HR}=2.71 ; 95 \% \mathrm{CI}=1.26-5.84 ; p=0.01)$.

Anti-tumor response

At the cut-off date, a response assessment was obtained for 140 patients. The best overall responses to osimertinib in the overall population included a CR of $3 \%(n=6)$, PR of $65 \%(n=102)$, SD of $14 \%(n=$ 22), and PD of $6 \%(\mathrm{n}=10)$, with an ORR of $68 \%$ and a DCR of $82 \%$. We further evaluated anti-tumor responses to osimertinib based on PD-L1 expression (Table 3). There was no statistically significant difference in the ORR among the high, low, and no TPS groups $(67 \%, 76 \%$, and $65 \%$, respectively; $p=$ $0.51)$. DCR was also similar among the high, low, and no TPS groups $(81 \%, 88 \%$, and $80 \%$, respectively; $p=0.57)$. 


\section{Discussion}

The present study investigated the clinical outcomes and the prognostic factors of osimertinib as a first-line treatment for advanced or recurrent NSCLC harboring EGFR mutations in a clinical practice setting. The results showed that the efficacy of osimertinib in the overall population was favorable. Although there was no statistically significant difference in the ORR among the high, low, and no TPS groups, the PFS of osimertinib in the high TPS group was inferior to that in the low or no TPS groups. Furthermore, univariate and multivariate analyses showed that high PD-L1 expression was an independent adverse prognostic factor associated with PFS in osimertinib treatment.

Compared to the FLAURA study, there were several differences in baseline patient characteristics in the present study. Specifically, in our study, there were more elderly patients and a higher frequency of decreased PS and presence of CNS metastases, while there was a lower frequency of never smokers. Racial differences were also observed. Some clinical factors such as decreased PS and the presence of CNS metastasis are poor prognostic factors for advanced NSCLC. Therefore, we conducted a subgroup analysis to evaluate whether these clinical factors affected the efficacy of osimertinib. The results showed that factors other than TPS did not affect the outcome (PFS) of osimertinib treatment. A previous phase II trial showed that osimertinib treatment provides a clinical benefit for EGFR T790M-mutated NSCLC whose PS score has declined to 2-4 [10]. In the FLAURA study, osimertinib resulted in significantly longer survival than the standard of care with first-generation EGFR-TKIs, even in patients 
who had CNS metastases at diagnosis [11]. Together with these previous reports, the present study indicates that osimertinib could be administered to such patients in clinical practice.

The estimated median PFS of osimertinib in the high TPS group was 10.1 months (95\% CI; 7.313.0), which was significantly shorter than that in the low or no TPS groups. Additionally, the present study showed that high TPS was an independent adverse factor associated with the PFS of osimertinib treatment. Several previous studies showed that $E G F R$-mutated advanced NSCLC with high TPS had poorer treatment outcomes with first- or second-generation EGFR-TKI compared with the weak or negative PD-L1 population $[12,13]$. Regarding osimertinib, a recent study of 71 patients who received first-line osimertinib revealed that patients with ind PD-L1 expression had poorer PFS than those with low or negative PD-L1 (median PFS, 5.0 vs. 17.4 months, $p<0.001$ ) [14]. The present study demonstrated similar results in a larger sample size. However, there was a discrepancy in the anti-tumor response between the previous and present studies. The previous study showed both ORR and DCR in the elevated PD-L1 group to be inferior to that in the low or negative PD-L1 group ( $p=0.043$ in ORR, and $p=0.006$ in DCR). In addition, the previous study also reported that the group with high PD-L1 expression had more frequent primary resistance to osimertinib compared with the group with low or no PD-L1 expression. In contrast, the present study showed no statistically significant difference in ORR and DCR regardless of PD-L1 expression ( $p=$ 0.51 in ORR and $p=0.57$ in DCR). The results of the present study suggest that once osimertinib showed favorable tumor shrinkage in the high TPS group similar with that in the low or no TPS group, it may 
become resistant in a short period thereafter. It remains controversial whether increased PD-L1 expression contributed to primary or acquired resistance to osimertinib treatment. Several preclinical studies showed that PD-L1 contributed to acquired resistance to EGFR-TKIs via the upregulation of Bcl-2-associated athanogene-1 and Yes-associated protein $1[15,16]$. Another preclinical study using EGFR-mutated NSCLC cell lines showed that PD-L1 is associated with primary resistance to EGFR-TKI by epithelialmesenchymal transition via the transforming growth factor- $\beta$ signal pathway [17]. Further basic research to reveal the molecular mechanism of the correlation between response to osimertinib and PD-L1 expression and clinical validation with a large cohort are warranted.

This study had some limitations. First, there was a bias originating from its retrospective nature. Second, OS was not reached owing to the short observation period. Third, information regarding TPS was not obtained in approximately $20 \%$ of participants. Finally, the current study included only Japanese patients; hence, ethnic differences may affect the results.

In conclusion, the current study provided clinically relevant data on the outcomes of first-line osimertinib for advanced or recurrent NSCLC with EGFR mutations. The favorable efficacy of osimertinib in this study was similar to that in the FLAURA trial. However, a high TPS could be an independent adverse prognostic factor for PFS in osimertinib therapy. 


\section{Acknowledgment}

We would like to acknowledge Editage (https://www.editage.jp) for English language correction.

\section{Funding}

The authors declare that no funding was received for conducting this study.

\section{Author information}

Toshihiro Shiozawa and Takeshi Numata contributed equally as co-first authors.

\section{Author contributions}

Toshihiro Shiozawa, Hiroaki Satoh, and Nobuyuki Hizawa planned and designed this study. Toshihiro

Shiozawa, Takeshi Numata, Tomohiro Tamura, Takeo Endo, Takayuki Kaburagi, Yusuke Yamamoto,

Hideo Ichimura, Hideyasu Yamada, Norihiro Kikuchi, Kazuhito Saito, Masaharu Inagaki, Koichi

Kurishima, Yasunori Funayama, Kunihiko Miyazaki, Nobuyuki Koyama, Kinya Furukawa, Hiroyuki

Nakamura, Shinji Kikuchi, Yukio Sato, Ikuno Sekine, and Hiroaki Satoh collected the data. Toshihiro

Shiozawa and Tomohiro Tamura analyzed the data. Toshihiro Shiozawa and Takeshi Numata wrote the

original draft. Nobuyuki Hizawa supervised the manuscript preparation. All authors discussed the results and agreed on the final draft. 


\section{Corresponding author}

Correspondence regarding this study to Toshihiro Shiozawa.

\section{Data availability}

Data concerning the results of this study are available from the corresponding author on reasonable request.

\section{Ethics declarations}

\section{Ethical approval}

This study was initiated after the study protocol was approved by the institutional review board of all institutions (approval number in Tsukuba University Hospital: R01-385). This retrospective observational study was conducted in compliance with the Helsinki Declaration. Individual patient data were anonymized prior to enrollment.

\section{Informed consent}

Informed consent was waived because the present study was a retrospective observational research. Optout was done on the website of each institution. 


\section{Competing interest}

There are no relevant competing interests to be disclosed. 


\section{References}

1. Jorissen RN, Walker F, Pouliot N, Garrett TP, Ward CW, et al. Epidermal growth factor receptor: mechanisms of activation and signalling. Exp Cell Res. 2003;284:31-53.

https://doi.org/10.1016/s0014-4827(02)00098-8

2. Maemondo M, Inoue A, Kobayashi K, Sugawara S, Oizumi S, Isobe H, et al. Gefitinib or chemotherapy for non-small-cell lung cancer with mutated EGFR. N Engl J Med. 2010;362:2380-8. https://doi.org/10.1056/NEJMoa0909530

3. Zhou C, Wu YL, Chen G, Feng J, Liu XQ, Wang C, et al. Erlotinib versus chemotherapy as first-line treatment for patients with advanced EGFR mutation-positive non-small-cell lung cancer (OPTIMAL, CTONG-0802): a multicentre, open-label, randomised, phase 3 study. Lancet Oncol. 2011;12:735-42. https://doi.org/10.1016/S1470-2045(11)70184-X

4. Sequist LV, Yang JC, Yamamoto N, O’Byrne K, Hirsh V, Mok T, et al. Phase III study of afatinib or cisplatin plus pemetrexed in patients with metastatic lung adenocarcinoma with EGFR mutations. J

Clin Oncol. 2013;31:3327-34. https://doi.org/10.1200/JCO.2012.44.2806

5. Soria JC, Ohe Y, Vansteenkiste J, Reungwetwattana T, Chewaskulyong B, Lee KH, et al. Osimertinib in untreated EGFR-mutated advanced non-small-cell lung cancer. N Engl J Med.

2018;378:113-25. https://doi.org/10.1056/NEJMoa1713137 
6. Ramalingam SS, Vansteenkiste J, Planchard D, Cho BC, Gray JE, Ohe Y, et al. Overall survival with osimertinib in untreated, EGFR-mutated advanced NSCLC. N Engl J Med. 2020;382:41-50. https://doi.org/10.1056/NEJMoa1913662

7. Hanna NH, Robinson AG, Temin S, Baker S Jr, Brahmer JR, Ellis PM, et al. Therapy for Stage IV non-small-cell lung cancer with driver alterations: ASCO and $\mathrm{OH}(\mathrm{CCO})$ joint guideline update. J Clin Oncol. 2021;39:1040-91. https://doi.org/10.1200/JCO.20.03570

8. Herbst RS, Baas P, Kim DW, Felip E, Pérez-Gracia JL, Han JY, et al. Pembrolizumab versus docetaxel for previously treated, PD-L1-positive, advanced non-small-cell lung cancer (KEYNOTE010): a randomised controlled trial. Lancet. 2016;387:1540-50. https://doi.org/10.1016/S0140$6736(15) 01281-7$

9. Reck M, Rodríguez-Abreu D, Robinson AG, Hui R, Csőszi T, Fülöp A, Gottfried M, et al. Pembrolizumab versus chemotherapy for PD-L1-positive non-small-cell lung cancer. N Engl J Med. 2016;375:1823-33. https://doi.org/10.1056/NEJMoa1606774

10. Nakashima K, Ozawa Y, Daga H, Imai H, Tamiya M, Tokito T, et al. Osimertinib for patients with poor performance status and EGFR T790M mutation-positive advanced non-small cell lung cancer: a phase II clinical trial. Investig New Drugs. 2020;38:1854-61. https://doi.org/10.1007/s10637-02000943-0 
11. Reungwetwattana T, Nakagawa K, Cho BC, Cobo M, Cho EK, Bertolini A, et al. CNS response to osimertinib versus standard epidermal growth factor receptor tyrosine kinase inhibitors in patients with untreated EGFR-mutated advanced non-small-cell lung cancer. J Clin Oncol.

2018;36:JCO2018783118. https://doi.org/10.1200/JCO.2018.78.3118

12. Hsu KH, Huang YH, Tseng JS, Chen KC, Ku WH, Su KY, et al. High PD-L1 expression correlates with primary resistance to EGFR-TKIs in treatment naïve advanced EGFR-mutant lung adenocarcinoma patients. Lung Cancer. 2019;127:37-43.

https://doi.org/10.1016/j.lungcan.2018.11.021

13. Su S, Dong ZY, Xie Z, Yan LX, Li YF, Su J, et al. Strong programmed death ligand 1 expression predicts poor response and de novo resistance to EGFR tyrosine kinase inhibitors among NSCLC patients with EGFR mutation. J Thorac Oncol. 2018;13:1668-75.

https://doi.org/10.1016/j.jtho.2018.07.016

14. Yoshimura A, Yamada T, Okuma Y, Fukuda A, Watanabe S, Nishioka N, et al. Impact of tumor programmed death ligand-1 expression on osimertinib efficacy in untreated EGFR-mutated advanced non-small cell lung cancer: a prospective observational study. Transl Lung Cancer Res. 2021;10:3582-93. https://doi.org/10.21037/tlcr-21-461 
15. Lin PL, Wu TC, Wu DW, Wang L, Chen CY, Lee H. An increase in BAG-1 by PD-L1 confers resistance to tyrosine kinase inhibitor in non-small cell lung cancer via persistent activation of ERK signalling. Eur J Cancer. 2017;85:95-105. https://doi.org/10.1016/j.ejca.2017.07.025

16. Tung JN, Lin PL, Wang YC, Wu DW, Chen CY, Lee H. PD-L1 confers resistance to EGFR mutation-independent tyrosine kinase inhibitors in non-small cell lung cancer via upregulation of YAP1 expression. Oncotarget. 2018;9:4637-46. https://doi.org/10.18632/oncotarget.23161

17. Zhang Y, Zeng Y, Liu T, Du W, Zhu J, Liu Z, et al. The canonical TGF- $\beta$ /Smad signalling pathway is involved in PD-L1-induced primary resistance to EGFR-TKIs in EGFR-mutant non-small-cell lung cancer. Respir Res. 2019;20:164. https://doi.org/10.1186/s12931-019-1137-4 
Table 1. Patient characteristics

\begin{tabular}{|c|c|}
\hline & $\mathrm{N}=158$ \\
\hline Age (years) & $73(39-93)$ \\
\hline \multicolumn{2}{|l|}{ Sex } \\
\hline Male & 66 \\
\hline Female & 92 \\
\hline \multicolumn{2}{|l|}{ Smoking status } \\
\hline Never & 83 \\
\hline Former or Current & 74 \\
\hline Unknown & 1 \\
\hline \multicolumn{2}{|l|}{ PS } \\
\hline 0/1/2/3/4/unknown & $50 / 79 / 18 / 7 / 2 / 2$ \\
\hline
\end{tabular}

Clinical stage

3/4/recurrent/other

$15 / 120 / 22 / 1$

Histology

Adenocarcinoma

Squamous cell carcinoma 5

Other 3

EGFR mutation

Exon 19 del $\quad 82$

$\begin{array}{ll}\mathrm{L} 858 \mathrm{R} & 68\end{array}$

$\begin{array}{ll}\text { Uncommon } & 8\end{array}$

CNS metastasis

Yes $\quad 45$

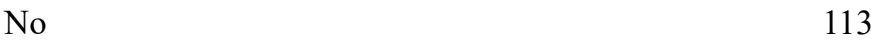

PD-L1 expression

None 60

Low 41

$\begin{array}{ll}\text { High } & 27\end{array}$

Unknown 30

Abbreviations: PS, performance status; EGFR; epidermal growth factor receptor; CNS, central nervous system; PD-L1, programmed death-ligand 1 
Table 2. Univariate and multivariate analyses of factors related to PFS

\begin{tabular}{|c|c|c|c|c|}
\hline \multirow[b]{2}{*}{ Variables } & \multicolumn{2}{|c|}{ Univariate } & \multicolumn{2}{|c|}{ Multivariate } \\
\hline & HR 95\% CI & $P$ value & HR 95\% CI & $p$-value \\
\hline \multicolumn{5}{|l|}{ Age } \\
\hline$<70$ years & Ref & & Ref & \\
\hline$\geq 70$ years & $0.73(0.37-1.44)$ & 0.36 & $1.00(0.47-2.11)$ & 0.99 \\
\hline \multicolumn{5}{|l|}{ Sex } \\
\hline Male & Ref & & Ref & \\
\hline Female & $1.56(0.79-3.50)$ & 0.20 & $1.58(.064-3.84)$ & 0.32 \\
\hline \multicolumn{5}{|l|}{ Smoking } \\
\hline Never & Ref & & Ref & \\
\hline Current or former & $1.06(0.54-2.07)$ & 0.87 & $0.98(0.39-2.45)$ & 0.96 \\
\hline \multicolumn{5}{|l|}{ PS } \\
\hline $0-1$ & Ref & & Ref & \\
\hline $2-4$ & $1.48(0.57-3.85)$ & 0.41 & $1.67(0.60-4.63)$ & 0.32 \\
\hline \multicolumn{5}{|l|}{ Stage } \\
\hline III or recurrent & Ref & & Ref & \\
\hline IV & $0.66(0.33-1.32)$ & 0.24 & $0.40(0.13-1.21)$ & 0.10 \\
\hline \multicolumn{5}{|l|}{ Mutation type } \\
\hline Exon 19 del & Ref & & Ref & \\
\hline L858R & $1.41(0.62-3.11)$ & 0.42 & $1.97(0.86-4.50)$ & 0.10 \\
\hline \multicolumn{5}{|l|}{ CNS metastasis } \\
\hline Absent & Ref & & Ref & \\
\hline Present & $1.18(0.57-2.43)$ & 0.65 & $1.20(0.53-2.68)$ & 0.66 \\
\hline \multicolumn{5}{|l|}{ TPS } \\
\hline None or low & Ref & & Ref & \\
\hline High & $2.37(1.16-4.83)$ & 0.02 & $2.71(1.26-5.84)$ & 0.01 \\
\hline
\end{tabular}

Abbreviations: PFS, progression-free survival; HR, hazard ratio; CI, confidence intervals; PS, performance status; CNS, central nervous system TPS, tumor proportion score; Ref, reference 
Table 3. Response assessment according to PD-L1 expression level

\begin{tabular}{lccc}
\hline & TPS high $(\mathrm{n}=27)$ & TPS low $(\mathrm{n}=41)$ & No TPS $(\mathrm{n}=60)$ \\
\hline Complete response (CR) & 0 & 1 & 2 \\
Partial response (PR) & 18 & 30 & 37 \\
Stable disease (SD) & 4 & 5 & 9 \\
Progressive disease (PD) & 2 & 2 & 4 \\
No evaluable & 3 & 3 & 8 \\
Objective response rate (ORR) & $67 \%$ & $76 \%$ & $65 \%$ \\
Disease control rate (DCR) & $81 \%$ & $88 \%$ & $80 \%$ \\
\hline
\end{tabular}

Abbreviations: TPS, tumor proportion score 


\section{Figure captions}

Fig 1. Progression-free survival according to age (a), sex (b), smoking status (c), PS (d), stage (e), mutation subtype (f), CNS metastasis (g), and PD-L1 expression levels (h).

Abbreviations: PS, performance status; CNS, central nervous system; PD-L1, programmed death-ligand 1;

CI, confidence interval; NR, not reached 
Figures

a

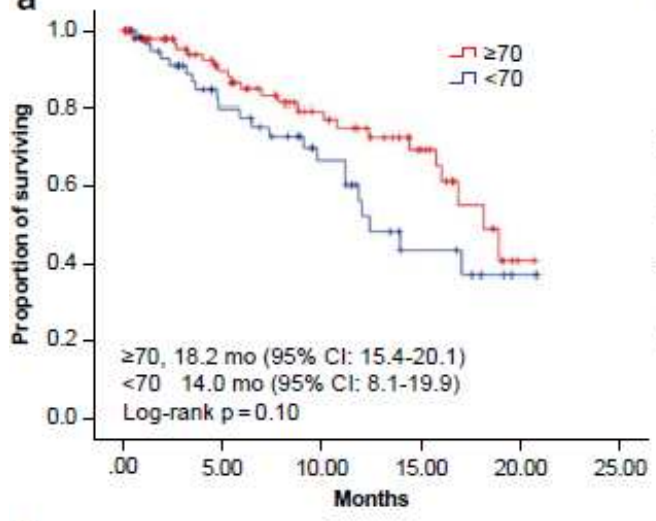

d

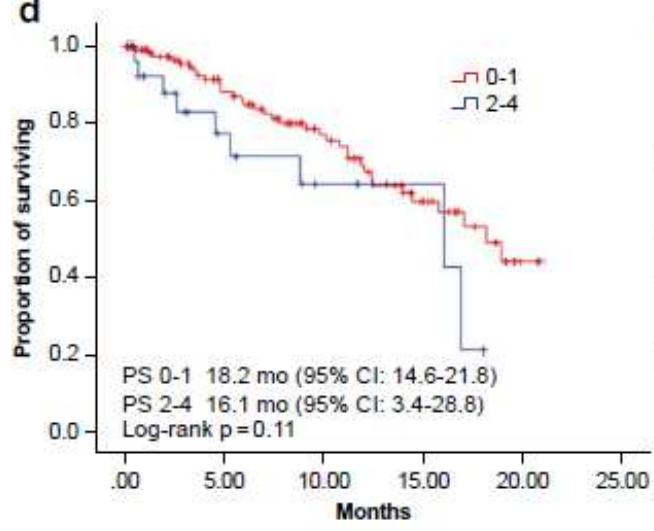

g

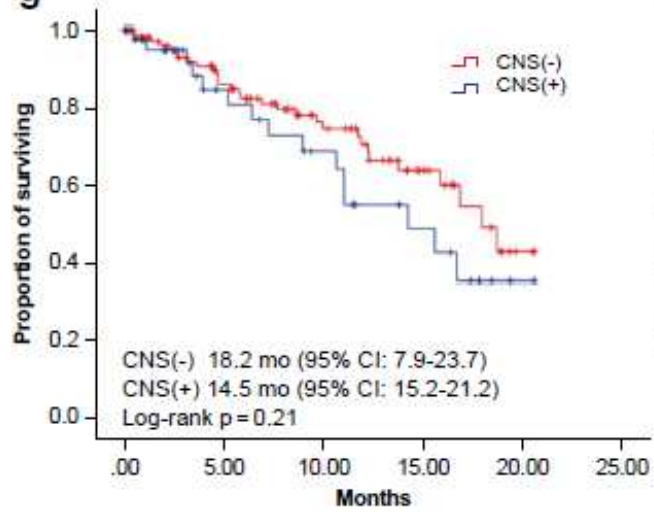

b

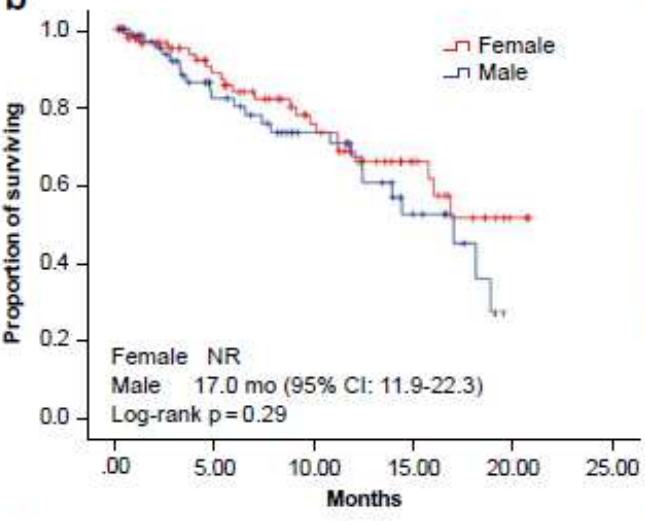

e

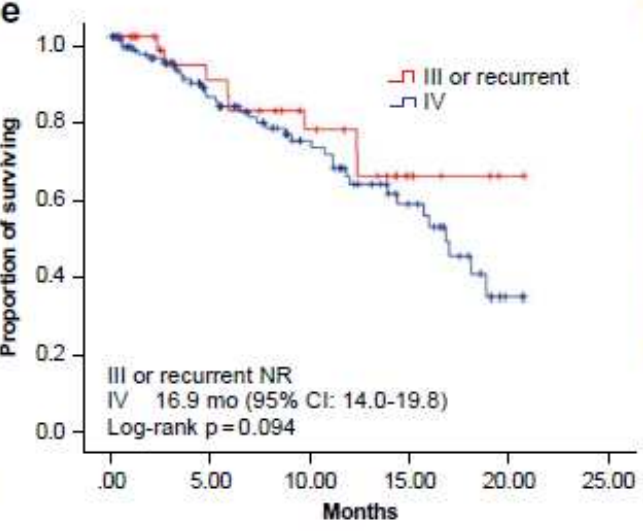

h

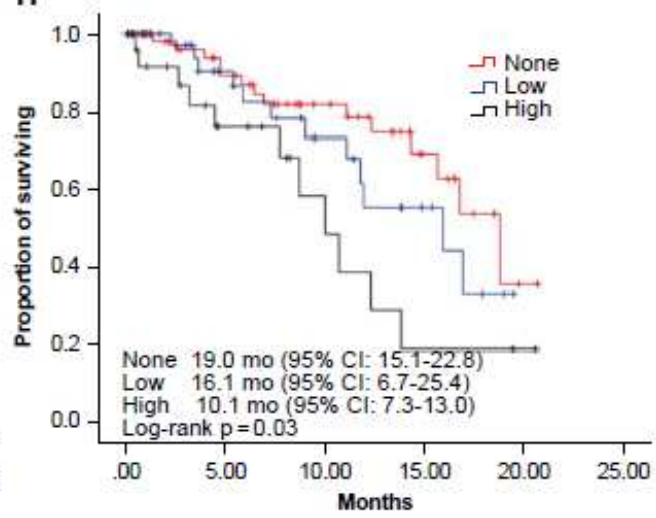

C

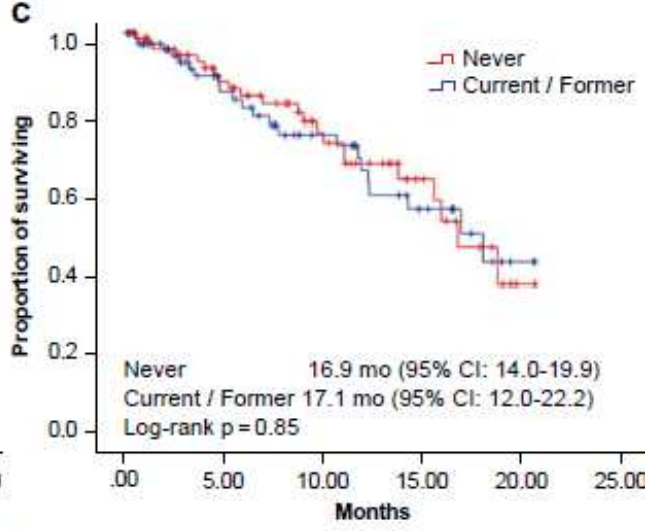

f

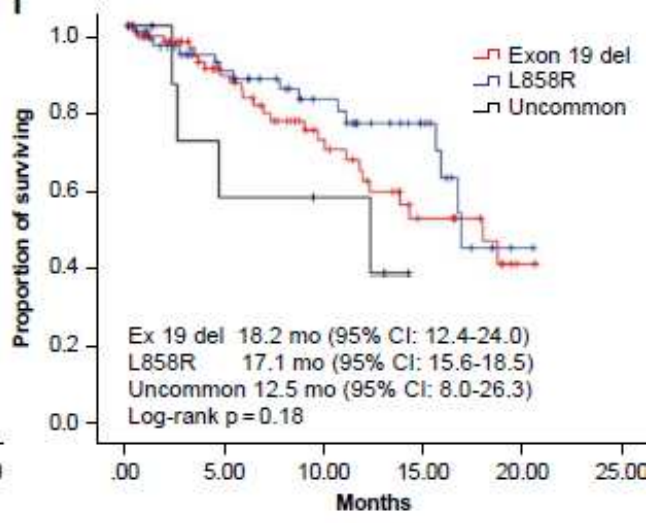

Figure 1

Progression-free survival according to age (a), sex (b), smoking status (c), PS (d), stage (e), mutation subtype (f), CNS metastasis (g), and PD-L1 expression levels (h).

Abbreviations: PS, performance status; CNS, central nervous system; PD-L1, programmed death-ligand 1; $\mathrm{Cl}$, confidence interval; NR, not reached 